Meta

Journal des tradlucteurs

Translators' Journal

\title{
De l'équivalence à l'adaptation
}

\section{Momar Khary Diagne}

Volume 16, numéro 3, septembre 1971

URI : https://id.erudit.org/iderudit/002563ar

DOI : https://doi.org/10.7202/002563ar

Aller au sommaire du numéro

Éditeur(s)

Les Presses de l'Université de Montréal

ISSN

0026-0452 (imprimé)

1492-1421 (numérique)

Découvrir la revue

Citer cet article

Diagne, M. K. (1971). De l'équivalence à l'adaptation. Meta, 16(3), 153-159.

https://doi.org/10.7202/002563ar d'utilisation que vous pouvez consulter en ligne.

https://apropos.erudit.org/fr/usagers/politique-dutilisation/ 


\title{
L'ÉQUIVALENCE \\ L'ADÄPTATION
}

\author{
"O wondrous power of words, by simple fact \\ Licensed to take the Meaning that we love." \\ "Dans le monde des mots, il faut s'incliner devant l'axiome. *
}

Vinay et Darbelnet ${ }^{1}$ définissent la MOdulation comme «le procédé par lequel on change de point de vue ». Dans sa «largeur», cette définition englobe l'ÉQUIVALENCE : "procédé de traduction qui rend compte de la même situation que l'original, en ayant recours à une rédaction entièrement différente », et l'ADAPTATION : «utilisation d'une équivalence reconnue entre deux situations ».

Si l'on conçoit la traduction comme la représentation de deux aspects d'une même réalité (égale à elle-même) en LD et en LA, dans quelle mesure l'équivalence et l'adaptation n'ont-elles pas droit de cité au sein d'une même famille linguistique ?

Considérant la famille francophone, plusieurs équivalences de situation ont donné naissance à toute une gamme d'expressions plus ou moins figées qui ne sauraient être tout simplement classées dans le fichier "série synonymique ».

Au même niveau de langue, "dès le lever du soleil » correspondra à « dès le chant du coq », «il fait un froid sibérien » à « il fait froid à pierre fendre», « il est de mauvaise humeur ce matin » à « il s'est levé du pied gauche »...

$\mathrm{Au}$ niveau de la conceptualisation, la schématisation linguistique d'une idée (de rapidité, de ruse, de longueur, etc.) sera affectée d'un paramètre différent selon le milieu socioculturel, le degré d'instruction du locuteur, etc. Dans les exemples choisis, il s'agira donc essentiellement de la langue parlée et il est évident que dans une même langue $X$, les idiolectes de $A, B, C .$. ne se plient pas à cette simplification. Exemples :

1. J. P. Vinay et J. Darbelnet, Stylistique comparée du français et de langlais, Paris, Didier, 1958. 


\begin{tabular}{|c|c|c|c|}
\hline Rapide & $\left\{\begin{array}{l}\text { comme une flèche (Fr.) } \\
\text { comme un épervier (Afr.) } \\
\text { comme l'éclair (Can.) }\end{array}\right.$ & Jaloux & $\left\{\begin{array}{l}\text { comme un pigeon ( } \mathrm{Fr} . \text {.) } \\
\text { comme un jeune marié (Afr.) } \\
\text { comme un pigeon (Can.) }\end{array}\right.$ \\
\hline Rusé & $\left\{\begin{array}{l}\text { comme un renard (Fr.) } \\
\text { comme un lièvre (Afr.) } \\
\text { comme un renard (Can.) }\end{array}\right.$ & Innocent & $\left\{\begin{array}{l}\text { comme un nouveau-né (Fr.) } \\
\text { comme un bébé (Afr.) } \\
\text { comme un enfant (Can.) }\end{array}\right.$ \\
\hline Long & $\left\{\begin{array}{l}\text { comme un jour sans pain (Fr.) } \\
\text { comme un serpent (Afr.) } \\
\text { comme une nuit d'hiver (Can.) }\end{array}\right.$ & Fier & $\left\{\begin{array}{l}\text { comme Artaban, un loup (Fr.) } \\
\text { comme un paon (Afr.) } \\
\text { comme Artaban, un loup (Can.) }\end{array}\right.$ \\
\hline Fort & $\left\{\begin{array}{l}\text { comme un turc, nègre (Fr.) } \\
\text { comme un lutteur, docker (Afr.) } \\
\text { comme un bœuf, cheval (Can.) }\end{array}\right.$ & Courageux & $\left\{\begin{array}{l}\text { comme un tirailleur (Fr.) } \\
\text { comme un lion (Afr.) } \\
\text { comme un lion (Can.) }\end{array}\right.$ \\
\hline Grand & $\left\{\begin{array}{l}\text { comme une girafe (Fr.) } \\
\text { comme un fromager (Afr.) } \\
\text { comme une girafe (Can.) }\end{array}\right.$ & Bavard & $\left\{\begin{array}{l}\text { comme une pie (Fr.) } \\
\text { comme un griot (Afr.) } \\
\text { comme une pie (Can.) }\end{array}\right.$ \\
\hline Paresseux & $\left\{\begin{array}{l}\text { comme un loir (Fr.) } \\
\text { comme une couleuvre (Afr.) } \\
\text { comme un lézard (Can.) }\end{array}\right.$ & Changeant & $\left\{\begin{array}{l}\text { comme les saisons (Fr.) } \\
\text { comme un caméléon (Afr.) } \\
\text { comme les saisons (Can.) }\end{array}\right.$ \\
\hline Propre & $\left\{\begin{array}{l}\text { comme un sou neuf (Fr.) } \\
\text { comme une ménagère (Afr.) } \\
\text { comme un chat (Can.) }\end{array}\right.$ & & \\
\hline
\end{tabular}

Dans la famille anglophone qui, géographiquement, est plus dispersée, les disparités de schèmes de comparaison semblent être plus nombreuses. Toutefois, considérant l'Angleterre et l'Amérique, « deux pays séparés par la même langue ${ }^{2}$ ", il a été possible de dégager ces quelques exemples, choisis arbitrairement :

$\begin{array}{ll}\text { Clean } & \left\{\begin{array}{l}\text { as a whistle (U.S.) } \\ \text { as a cat (U.K.) }\end{array}\right. \\ \text { Proud } & \left\{\begin{array}{l}\text { as Punch (U.S.) } \\ \text { as a peacock (U.K.) }\end{array}\right. \\ \text { Slow } & \left\{\begin{array}{l}\text { as a tortoise (U.S.) } \\ \text { as a snail (U.K.) }\end{array}\right. \\ \begin{array}{r}\text { Sharp- } \\ \text { eyed }\end{array} & \left\{\begin{array}{l}\text { as a hawk (U.S.) } \\ \text { as a sailor (U.K.) }\end{array}\right. \\ \text { Quick } & \left\{\begin{array}{l}\text { as lightning (U.S.) } \\ \text { before you could say } \\ \text { Jack Robinson (U.K.) }\end{array}\right.\end{array}$

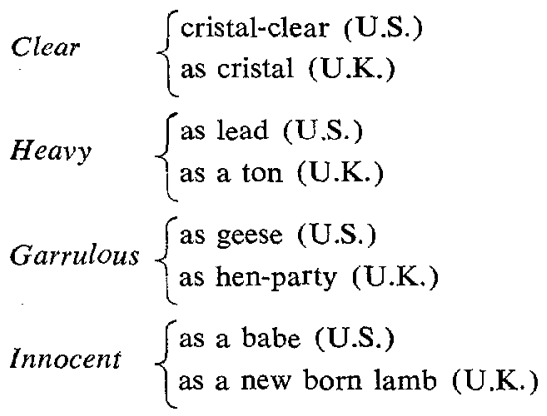

On le voit, beaucoup de ces paramètres se recoupent dans la démarche conceptuelle. Au demeurant, certains d'entre eux, figés par l'usage en expressions consacrées, servent davantage de notions de référence que de schèmes de comparaison : before you could say Jack Robinson indique la notion de rapidité outreAtlantique, tandis que quick as lightning en exprime l'idée. Dans le domaine de l'adaptation, le célèbre exemple de la parabole du figuier de E. Nida devra être transposé en parabole du «tamarinier», par exemple, pour être assimilé par les autochtones de telle région évangélisée d'Afrique; raison sans doute pour

2. De Bernard Shaw. 
laquelle l'abbé Boilat ${ }^{3}$ a trouvé si ardue la traduction des Écritures en Wolof. De la même manière, «tu seras blanc comme neige » pour symboliser l'absence de péchés du prosélyte pourra être adapté comme suit : «tu seras blanc comme du kapok, du coton » ou chez des peuples où le jaune ou le safran est associé à la chasteté, à la pureté, «tu seras jaune comme un champ de maïs pendant l'hivernage ». Le génie de la langue française a quelque peu négligé de telles «adaptations»: après tout, les «cimes du Canigou» ne sont pas plus belles que les hauteurs du Fouta-Djalon.

Ainsi donc, l'équivalence comme l'adaptation peuvent s'opérer dans une même langue (LD) où il y a, en principe, identité de cheminement linguistique. Est-ce à dire que vers une LA où prédomine, dans le rapport avec la $L D$, une certaine discordance entre les expériences, les attitudes et les valeurs, toute la traduction n'est que matière à équivalences et adaptations ?

L'équivalence participant de la même démarche que la modulation, à savoir un changement de point de vue, de perspective, isole la situation dans le contexte du texte comme dans celui de la langue. Exemple :

When/young Dr. Sargent arrived at the college/to assume his duties/as a member of the faculty, /he reversed a lifelong custom.

Ce matin-là, /le professeur Sargent arriva au collège/pour assumer ses fonctions/comme membre du corps professoral/et comprit qu'il n'était plus du même côté de la barrière./

L'équivalence réside ici dans le fait que le texte fait comprendre que le professeur en question a jusque-là été simple étudiant. Au niveau de la langue, "la barrière » sépare depuis toujours l'enseignant de ses élèves. Elle est assimilable à une frontière, que le jeune professeur vient de franchir. À la limite, «barrière» rejoint le cliché souligné par Vinay et Darbelnet du «témoin appelé à la barre » par un Perry Mason disant : «Will the defendant go to the witness-box», comme si le professeur Sargent était dans le «box » des enseignants. L'équivalence peut aussi exploiter un côté de la langue d'arrivée soulignant un trait, un aspect particulier des locuteurs de cette même langue. Exemple :

Their town houses are nothing but places to pour : Leurs maisons de ville sont des piedhang their hats. à-terre.

Cette équivalence fait allusion à une situation connue : à savoir que les Anglais et les Londoniens, car c'est d'eux que P. Morand parle ici, qui portent des couvre-chefs plus souvent que les Français, utilisent, aux yeux du traducteur français, leurs pied-à-terre plus comme «porte-chapeaux » que comme autre chose. La force de l'allusion n'impose pas ipso facto l'emploi de telle équivalence plutôt que de telle autre. Autrement dit, l'équivalence ne relève pas nécessairement du cliché «suffisamment typique pour être reconnu de prime abord» ou de l'allusion prestigieuse.

Dans cette optique, l'équivalence, dans sa mise en valeur accentuelle d'une partie du message, participe de la transposition. C'est le cas dans bien des maximes et proverbes dont la valeur de pensée prime la valeur syntactique. Il est alors superflu de chercher si tel mot a changé de catégorie grammaticale ou si tel autre s'est «perdu» dans la traduction. Le message devient «module» de pensée.

3. Abbé de Saint-Louis du Sénégal, à l'époque coloniale. 
Exemples :

Birds of a feather make a flock together.

Qui se ressemble s'assemble.

The sun will shine on those who stand before Le premier levé, le premier servi.

it does on those who kneel under them ${ }^{4}$.

It all depends whose $\mathrm{cx}$ is gored.

Every cloud has a silver lining.

Tout dépend des intérêts en jeu.

Après la pluie, le beau temps.

Cependant, la vie moderne n'est pas faite de «sagesse », mais de concepts linguistiques qui reflètent la complexité des problèmes humains. Dans quelle mesure l'équivalence contribue-t-elle alors à restreindre le halo qui se forme au niveau de la «symbolique » d'un syntagme en LD ? Considérons par exemple la phrase : He does look ill, does'nt he ?, le syntagme verbal look ill se refère à l'apparence "malade» de la personne en question autant qu'à sa "mauvaise mine», anormalement triste. L'équivalence : «Il a vraiment mauvaise mine, n'est-ce pas?» couvre l'aspect « humeur» autant que l'aspect «maladie». Il en est de même de : one way street $=$ sens interdit $;$ hang-over $=$ gueule de bois ; point counter point $=$ contrepoint. L'équivalence connaît aussi, avec le développement technologique des temps modernes, une croissance parallèle, spécialement dans les domaines techniques. Ici, la tendance à l'abréviation pour désigner par un sigle un mécanisme électronique, une innovation technique ou tout simplement une pratique dont l'anglais (et surtout l'américain) est friand, semble gagner de plus en plus le français « universel ». Exemples :

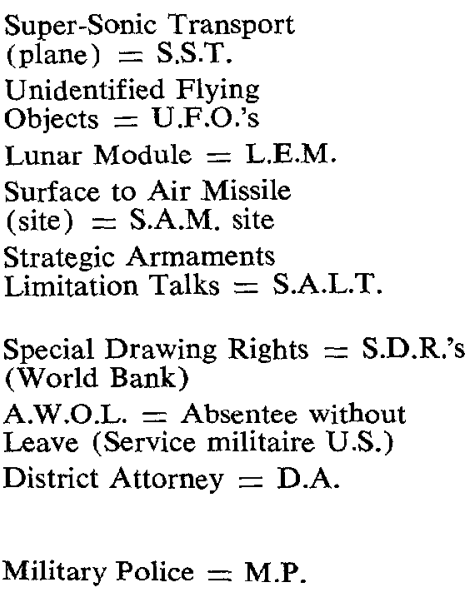

T.S.S. = Transport supersonique

S.V. = Soucoupes volantes

Lem $=$ module lunaire

Fusée SAM en silo, ce qui est tautologique.

Après Helsinki, les conversations $S A L T$ ont repris hier à Vienne 5 . Là aussi il y a tautologie.

"Or-papier " = droits de tirage spéciaux.

Désertion temporaire : il est en «D.T, »

Procureur (général) de la république, (que je n'ai pas encore vu abrégé en P.g.R. ou en P.R.)

Étant donné que P.M. est aussi l'abréviation de Préparation militaire, on a plus souvent recours à l'emprunt «M.P."

Dans ces cas, l'équivalence est en marge de l'emprunt et du calque, et, dans la mesure où elle est vulgarisée partout dans le monde du français universel par des romans policiers à la San Antonio et par des journaux et revues qui se veulent «ouverts sur le monde anglo-saxon» (sic), elle aura de fil en aiguille droit de cité, à l'encontre des efforts d'Etiemble et de l'Académie, dans la langue parlée et écrite des francophones et des écrivains francophones; et par le biais des

4. Citation extraite de Things fall apart de Chinua Achebe. Le Britannique dit plus souvent : "The early bird gets the worm."

5. L'exemple 5 est extrait de la Presse du 6 avril 1970 (Section : Nouvelles internationales). 
domaines où le français accuse des lacunes indubitables : le sport, la marine, la technique et le scientifique, les affaires. RADAR (Radio Detecting and Ranging), qui est un emprunt, aurait pu avoir un équivalent français, peut-être moins assonant, mais conforme au génie (en tant que pouvoir de forger des vocables et non comme «mode de pensée ») du français universel. Il faut d'abord "oser» former le néologisme «Système de détection radio-électrique» avant de savoir si SYDREL * est «français » et de laisser l'Académie française épiloguer sur le problème.

Autrement dit, l'empruntomanie du français universel de 1970 nuit grandement à l'établissement d'équivalences qui pourraient être reconnues universellement.

C'est ici que l'ADAPTATION peut jouer un rôle capital dans la métalinguistique de la traduction. D'emblée, l'adaptation s'efforce de combler un «vide » de traduction, un halo conceptuel, en opérant une restructuration complète de la syntaxe de la LA ; tout en gardant l'esprit (donc l'essence du message) du texte de la LD. À la limite, on pourrait postuler, paraphrasant M. McLuhan, que dans l'adaptation le "médium », c'est-à-dire le signifiant, n'est pas le «message », c'est-à-dire le signifié.

C'est sans doute pour cette raison que le terrain de prédilection de l'adaptation a traditionnellement été le domaine littéraire. Après le succès qu'a connu la traduction de Poe par Baudelaire et Mallarmé, qui a contribué à redorer le blason du poète américain, après l'excellente transposition de If de Kipling par l'angliciste chevronné que fut Maurois, Gide, par un « acte gratuit», s'est proposé de rendre Shakespeare, après tant d'autres écrivains-traducteurs. À la scène I de l'acte $\mathrm{V}$ de Hamlet, il propose ceci :

Le plus grand des Césars/n'est plus qu'un peu de cendre/

Et celui qui/faisait trembler tout l'univers/

Au plus modeste emploi/nous le voyons descendre :/

Boucher un trou/par où/souffle le vent d'hiver.

pour rendre :

Imperious Caesar,/dead and turn'd to clay/

Might stop a hole/to keep the wind away :

O! that-that earth,/which kept the world in awe,/

Should patch a wall/to expell the winter's flow./

Les unités de traduction (UT) se correspondent : 8 en LA et 8 en LD. L'ordre des mots a été savamment bouleversé et plusieurs syntagmes ont été dilués et explicités dans le processus d'adaptation. En LD : UT 3 devient UT 7 en LA Might $=$ au plus modeste emploi. En LD : UT 4 devient UT 8 en LA Should $=$ nous le voyons descendre. L'adaptation proprement dite se situe au niveau des restes de César que l'auteur anglais considère comme de l'«argile »: UT 2, et que le traducteur français dénomme, très justement : «les cendres de... », cendre, modulé négativement : «qu'un peu de cendre», et du niveau de la gloire du défunt empereur : UT 6 en LD = UT 4 en LA.

Cependant, cette forme d'adaptation est devenue plus ou moins synonyme d' « acclimatation» d'auteurs qui se sont fait une place au soleil de la littérature universelle, au sens catholique du terme : Shakespeare, Molière, Goethe... Imper-

6. SDN et $\mathrm{OCDE}$ sont conformes au génie du français.

7. Ousmane Sembène, romancier et cinéaste sénégalais. 
ceptiblement, elle a glissé vers les romans de poche (cheap novels), d'espionnage ou d'amour, selon la latitude, qui se réclament d'un certain type de héros à vocation universaliste (James Bond 007 et consorts) à la faveur de la mondialisation du cinéma hollywoodien. De même que les classiques ont été traduits en plusieurs langues, de même ces «néo-modernes » qui ont nom Ian Fleming, Jean Bruce et Agatha Christie sont traduits et «adaptés » en des myriades de langues et de langages dont... l'esperanto.

Par-delà cette forme vulgaire de l'adaptation, subsiste celle qui décortique le message sous l'angle culturel pour expliciter en LA ce que la LD sous-entend. Exemple :

Des automobiles basses et rapides,/véritables petits avions, /qui prennent jour par des hublots, / projettent chaque samedi,/dans toutes les directions,/d'abord vers les banlieues/qui voudraient créer l'illusion de la nature,/avec leurs architectures rustiques, puis vers la grande et vraie campagne,/des couples désormais sans enfants.

adapté comme suit :

...and every Saturday/they leave the children with Nanny/and take off in low-slung cars with discreet little windows,/fanning out in all directions/into the open country...,/bowling along through/endless suburbs full of homes/designed to bring a taste of the countryside within commuting distance of Town,.../

Dans cette phrase, plusieurs UT ont été adaptées en LA :

UT 2 : Nanny représente une adaptation de UT $9:$ \& des couples désormais sans enfants», qui aurait pu être interprétée comme «des couples sans enfants» en fait; «désormais» a été dilué et adapté en Nanny : qui aurait pu être Mammy dans le Sud des U.S.A. (en parlant d'une nourrice noire), Nounou dans certaines régions de France (le Midi), etc.

UT 3 : adapte et concentre UT 1, UT 2 et UT 3, l'aspect descriptif de hublots étant rendu par le couplet d'adjectifs discreet et little se rapportant à windows.

UT 4 : module UT 4 et UT 5, et le rejet «vers la vraie et grande campagne » est adapté par l'incidente into the open country.

UT 9 : adapte UT 7 de telle sorte que l'effort «volontaire» de changer le « décor urbain $\gg$ de Londres est explicité par designed to = qui voudraient créer [l'illusion] par a taste of the countryside $=$ l'illusion de la nature. Dès lors la toute dernière unité de LA représente un gain.

Donc, l'effort descriptif qui accompagne l'adaptation perturbe l'ordre syntaxique de la phrase qu'il restructure pour imprimer un aspect dynamique au message.

Ainsi :

It was vital and alive, an artery rushing with Tout ce monde était plein de vie, comme une blood. vraie fourmilière.

C'est ici que se situe la différence fondamentale entre l'équivalence et l'adaptation. Alors que la première module le signifié de l'enveloppe syntactique par un «tour» plus ou moins consacré par l'expérience vécue, donc éprouvée des «situations» (au sens presque sartrien du terme) humaines, la seconde, elle, se borne à remodeler le signifiant pour en sortir, au péril d'enchevêtrer la tournure grammaticale, l'ultima ratio du message. 
Par conséquent, l'adaptation est moins fidèle au message que l'équivalence. Mais il se présente des cas où celui-ci est rendu par une situation équivalente sans pour autant que l'ordre syntactique soit dérangé. Exemple :

Therefore/it is important/for us to understand/that there came a time/in the nineteenth century/when England departed from her historic policy/and when her strategy was modified/ to suit a brummagen imperialism.

Il est donc important/de comprendre qu'/il est venu un moment/au cours du dix-neuvième siècle/où l'Angleterre a rompu avec sa politique traditionnelle/et où sa stratégie a été modifiée/pour répondre aux vues d'un impérialisme de mauvais aloil.

Ici, toutes les UT se conservent, et dans la dernière, l'adaptation \& de mauvais aloi » induit brummagen : qui s'appliquait à la monnaie falsifiée de Birmingham au Xvir siècle.

Par-delà les phénomènes plus ou moins systématisables d'identification et de promotion des équivalences et des adaptations, il y a la dualité de la langue écrite et de la langue parlée... et l'ambiguité de la norme.

La première, en ce qui concerne le français, tend à être édictée par Paris et protégée par l'Académie française. La seconde est souvent imposée par Paris et réfutée ensuite par la même Académie. Il ne fait pas de doute que les réalités et les situations ne sont pas les mêmes partout dans le monde francophone. Le besoin «français » de les codifier ne doit donc pas signifier la non-acceptation de leur disparité, de leur valeur. Il est vrai que le français n'a pas le même statut patrout dans le monde de cette «francophonie» de l'heure : ici, il est langue de culture, là, langue de circonstance, là-bas, langue de civilisation. C'est sans doute la raison pour laquelle le « je suis là, seulement» des lycées du Sénégal, serait difficilement équivalent, pour le Québecois, à «ça va pas pire», pour le Marseillais, à «ça roule ». L'effort à accomplir à ce niveau relève de l'équivalence, effort que l'anglais semble faire à priori, plus souvent que le français. C'est aussi la raison pour laquelle ces «structures de surface» tendent à se niveler à l'échelle de la langue écrite : les écrits de Camus, de Ducharme, de Sembène accusent moins de disparités que les parlers méridional, gaspésien, goréen ${ }^{8}$. Mais, dans la mesure où le style est l'écart par rapport à la norme, les ceuvres de Troyat, de Godbout et de Dadié ${ }^{9}$, au sein du système linguistique du français universel, sont moins susceptibles d'être adaptées, dans une traduction vers une LA donnée, que celles de Mailer, de Maugham et d'Achebe ${ }^{10}$. La cause profonde de cette différence, E. Sapir la donne quand il écrit : "The drift of language is not properly concerned with changes of content at all, merely with changes in formal expression. »

La traduction, en descendant de son piédestal littéraire traditionnel pour se mettre au diapason du quotidien de la vie moderne, dominée par les pressions qu'exercent les progrès techniques - domaine où l'Amérique du Nord anglosaxonne excelle - mettra à la disposition de l'homme des outils de "conversation » (to communicate, en anglais) qui, à la faveur d'une civilisation de l'universel en devenir, seront moins sujets à ADAPTATION (les situations s'harmonisant), plus Soumis à l'ÉQUIVADENCE (les modes de penser subsistant). Dans cette perspective, le Canada francophone occupe une place de choix...

8. Gorée : île au large de Dakar.

MOMAR KHaRy Diagne

9. Bernard Dadié, écrivain et éducateur ivoirien.

10. Chinua Achebe, romancier nigérien. 